\title{
Erratum to: Determination of the geographical origin of Brazilian wines by isotope and mineral analysis
}

\author{
S. V. Dutra • L. Adami • A. R. Marcon • G. J. Carnieli • \\ C. A. Roani - F. R. Spinelli • S. Leonardelli • C. Ducatti • \\ M. Z. Moreira $\cdot$ R. Vanderlinde
}

Published online: 30 July 2011

(C) Springer-Verlag 2011

\section{Erratum to: Anal Bioanal Chem \\ DOI 10.1007/s00216-011-5181-2}

Unfortunately, there was a mistake in Table 1 of this contribution. Please find the correct Table 1 below.

Table 1 Isotope ratios ${ }^{18} \mathrm{O} /{ }^{16} \mathrm{O}$ (per mille) of water and ${ }^{13} \mathrm{C} /{ }^{12} \mathrm{C}$ (per mille) of ethanol of Cabernet Sauvignon and Merlot wines of the different regions studied

\begin{tabular}{|c|c|c|c|c|c|c|c|c|c|c|c|c|}
\hline \multirow[t]{2}{*}{ Regions } & \multicolumn{6}{|c|}{${ }^{18} \mathrm{O} /{ }^{16} \mathrm{O}(\% ;$ mean \pm standard error $)$} & \multicolumn{6}{|c|}{${ }^{13} \mathrm{C} /{ }^{12} \mathrm{C}(\% ;$ mean \pm standard error $)$} \\
\hline & Merlot & & $\begin{array}{l}\text { Cabernet } \\
\text { Sauvignor }\end{array}$ & & Total & & Merlot & & $\begin{array}{l}\text { Cabernet } \\
\text { Sauvignon }\end{array}$ & & Total & \\
\hline Serra Gaúcha & $0.38 \mathrm{Aa}$ & \pm 0.15 & $-0.28 \mathrm{Aa}$ & \pm 0.18 & $0.05 \mathrm{~A}$ & \pm 0.12 & $-27.73 \mathrm{Aa}$ & \pm 0.06 & $-28.52 \mathrm{Ab}$ & \pm 0.08 & $-28.12 \mathrm{~A}$ & \pm 0.06 \\
\hline Serra do Sudeste & $-2.34 \mathrm{Ca}$ & \pm 0.21 & $-2.17 \mathrm{Ca}$ & \pm 0.25 & $-2.28 \mathrm{C}$ & \pm 0.16 & $-28.75 \mathrm{Ba}$ & \pm 0.15 & $-29.82 \mathrm{Bb}$ & \pm 0.3 & $-29.11 \mathrm{~B}$ & \pm 0.18 \\
\hline Campanha & $-1.35 \mathrm{Ba}$ & \pm 0.39 & $-1.24 \mathrm{Ba}$ & \pm 0.3 & $-1.29 \mathrm{~B}$ & \pm 0.24 & $-28.90 \mathrm{Ba}$ & \pm 0.13 & $-29.37 \mathrm{Bb}$ & \pm 0.11 & $-29.17 \mathrm{~B}$ & \pm 0.09 \\
\hline Total & -0.23 & \pm 0.16 & -0.64 & \pm 0.15 & -0.44 & \pm 0.11 & $-28.08 \mathrm{a}$ & \pm 0.07 & $-28.82 b$ & \pm 0.07 & -28.45 & \pm 0.05 \\
\hline
\end{tabular}

Means followed by different capital letters in the column and means followed by different lowercase letters in the line differ significantly by ANOVA complemented by Tukey's test of multiple comparisons; significance level of 5\%

The online version of the original article can be found at http://dx.doi. org/10.1007/s00216-011-5181-2.

S. V. Dutra $\cdot$ R. Vanderlinde

Institute of Biotechnology, University of Caxias do Sul (UCS),

Rua Francisco Getúlio Vargas, 1130,

Caxias do Sul, Rio Grande do Sul 95070-560, Brazil

S. V. Dutra $(\bowtie) \cdot$ L. Adami $\cdot$ A. R. Marcon $\cdot$ G. J. Carnieli $\cdot$ C. A. Roani $\cdot$ F. R. Spinelli $\cdot$ S. Leonardelli $\cdot$ R. Vanderlinde IBRAVIN/LAREN - Reference Laboratory Enologic, Avenida da Vindima, 1855,

Caxias do Sul, Rio Grande do Sul 95084-470, Brazil

e-mail: scdutra@terra.com.br
C. Ducatti

Institute of Biosciences, Department of Physics and Biophysics, Centre for Stable Isotopes in Life Environmental Sciences,

Universidade Estadual Paulista Júlio de Mesquita Filho,

Rubião Júnior,

18608-000 Botucatu, São Paulo, Brazil

M. Z. Moreira

Center for Nuclear Energy in Agriculture,

Laboratory of Isotope Ecology, University of São Paulo,

Avenida Centenário, 303 São Dimas,

13400-970 Piracicaba, São Paulo, Brazil 